\title{
Multi-environment phenotyping of $C$. elegans for robust evaluation of physical performance
}

Jennifer E. Hewitt ${ }^{1,2}$, Ricardo Laranjeiro ${ }^{3}$, Masoud Norouzi ${ }^{1}$, Rebecca Ellwood ${ }^{4}$, Adam Antebi ${ }^{2}$, Nathaniel J. Szewczyk ${ }^{4}$, Monica Driscoll ${ }^{3}$, and Siva A. Vanapalli ${ }^{1}$

1. Department of Chemical Engineering, Texas Tech University, Lubbock, TX, USA

2. Department of Molecular Genetics of Ageing, Max Planck Institute for Biology of Ageing, and Cologne Excellence Cluster on Cellular Stress Responses in Aging-Associated Diseases (CECAD), University of Cologne, Cologne, Germany

3. Department of Molecular Biology and Biochemistry, Rutgers, The State University of New Jersey, Piscataway, NJ, USA

4. MRC/Arthritis Research UK Centre for Musculoskeletal Ageing Research, University of Nottingham, United Kingdom \& National Institute for Health Research Nottingham Biomedical Research Centre, Derby, UK 


\section{ABSTRACT}

Determining the physical performance of humans using several measures is essential to evaluating the severity of diseases, understanding the role of environmental factors, and developing therapeutic interventions. Development of analogous measures of physical performance in model organisms can help in identifying conserved signaling pathways and prioritizing drug candidates. In this study, we propose a multi-environment phenotyping (MEP) approach that generates a comprehensive set of measures indicative of physical performance in C. elegans. We challenge C. elegans in different mechanical environments of burrowing, swimming, and crawling, each of which places different physiological demands on the animals to generate locomotory forces. Implementation of the MEP approach is done using three established assays corresponding to each environment-a hydrogel-based burrowing assay, the CeleST swim assay, and the NemaFlex crawling strength assay. Using this approach, we study individuals and show that these three assays report on unique aspects of nematode physiology, as phenotypic measures obtained from different environments do not correlate with one another. Analysis of a subset of genes representative of oxidative stress, glucose metabolism, and fat metabolism show differential expression depending on the animal's environment, suggesting that each environment evokes a response with distinct genetic requirements. To demonstrate the utility of the MEP platform, we evaluate the response of a muscular dystrophy model of $C$. elegans dys-1 to drug interventions of prednisone, melatonin and serotonin. We find that prednisone, which is the current treatment standard for human Duchenne muscular dystrophy, confers benefits in all three assays. Furthermore, while the tested compounds improve the physical performance of $d y s-1$, these compounds are not able to fully restore the measures to 
wild-type levels, suggesting the need for discovery efforts to identify more efficacious compounds that could be aided using the MEP platform. In summary, the MEP platform's ability to robustly define C. elegans locomotory phenotypes demonstrates the utility of the MEP approach toward identification of candidates for therapeutic intervention, especially in disease models in which the neuromuscular performance is impaired. 


\section{INTRODUCTION}

2 Physical performance or fitness in humans is formally assessed using health-related or

3 skill-related measures [1]. Health-related measures include cardiorespiratory endurance, muscle

4 endurance, muscle strength, body composition, and flexibility. Skill-related measures include

5 agility, balance, coordination, power, speed and reaction time. Assessment of human physical

6 performance utilizing such measures is central to evaluating severity of diseases, especially

7 diseases in which the neuromuscular system is involved [2-4]. Likewise, declines in physical

8 performance have been shown to correlate with the risk of disability, hospitalization rates, and

9 mortality in the elderly [5-8].

10 Linking the molecular, cellular, and tissue-level mechanisms to human physical performance is

11 best achieved in model organisms as they allow identification of conserved signaling pathways

12 and therapeutic interventions. The nematode Caenorhabditis elegans is a popular model

13 organism that features relatively simple and well-understood biology, rapid lifecycle, and ease of

14 culture [9]. Importantly, genetic conservation supports the relevance of the C. elegans model for

15 studying gene activities and pathways relevant to a variety of human diseases [10-16] including

16 Duchenne muscular dystrophy [17], neurodegenerative diseases [18-20], and sarcopenia [21].

17 Moreover, C. elegans has a conserved muscular architecture comprising contractile elements and

18 attachments similar to higher animals. Thus, development of approaches to assess the physical

19 performance of C. elegans using measures that are analogous to human measures may help in

20 translating discoveries made in C. elegans to human health. 
21 Assessment of physical performance in the nematode C. elegans usually involves monitoring the

22 locomotion of crawling C. elegans on agar plates or thrashing in liquid [22, 23], although more

23 recently, assays have also been developed to characterize the burrowing ability of C. elegans [24,

24 25]. Technical advances in computer vision and data-driven approaches have led to increasing

25 the depth of phenotypic information from crawling and swim assays by generating

26 multidimensional readouts [26, 27]. Despite these significant advances, current approaches to

27 characterize C. elegans physical performance commonly rely on investigating animal

28 locomotory response in a singular mechanical environment, limiting the extent of physiological

29 and clinically relevant measures that can be obtained.

Depending on the mechanical resistance of the environment, C. elegans can adjust its gait and

31 body mechanics [28]. On agar plates, the surface tension of the thin liquid layer at the agar

32 surface constrains the animal motion to two dimensions and also aids in propulsive thrust [29]. In

33 liquid, the nematode body experiences rotational slip and therefore to effectively swim, the

34 internal forces generated within the animal need to balance the external hydrodynamic forces

35 [30]. In gel environments, the animal burrows by executing three-dimensional maneuvers,

36 making the neuromuscular actuation different from that on two-dimensional substrates [30, 31$]$.

37 Finally, studies have shown that in microfluidic pillar environments, animals can adjust body

38 gait, speed, and force generation depending on the geometry and mechanical resistance of the

39 pillar arena [32-34]. Thus, opportunities exist to expose C. elegans to different mechanical

40 environments and extract information analogous to human health-related or skill-related

41 measures. 
42 In this study, we establish a multi-environment phenotyping (MEP) platform that evaluates the

43 physical performance of C. elegans in three distinct mechanical environments. Our approach

44 combines three assays of physical performance — NemaFlex [34], CeleST [35], and a hydrogel-

45 based burrowing assay [25] — to assess animal physical capacities in environments that elicit

46 locomotory modes of crawling, swimming, and burrowing, respectively. By assaying the same

47 individual animal in each environment, we show that each assay provides distinct information on

48 animal capabilities, and that crawling, swimming, and burrowing evoke distinct responses

49 associated with changes in expression of different genes. To demonstrate the applicability of the

50 MEP platform for evaluating C. elegans disease models and therapeutic interventions, we assess

51 the physiological health of $d y s-1$ mutants, the C. elegans model for Duchenne muscular

52 dystrophy. We show that while the tested compounds improve $d y s-1$ health, the treatments are

53 not able to fully restore wild-type behaviors, underscoring the need for further discovery efforts

54 for compounds that counter defects in all the measures reported in the MEP platform.

\section{RESULTS}

\section{Overview of the multi-environment phenotyping approach}

58 The multi-environment phenotyping (MEP) platform consists of three assays conducted in

59 different mechanical environments that evoke one of three forms of locomotion from C. elegans:

60 burrowing, swimming, or crawling. We extracted descriptive measures from each of the three

61 environments (Figure 1). 
62 In the hydrogel burrowing assay [25] we load animals into the base of a well and add a layer of

63 Pluronic F-127 solution, which is a transparent and biocompatible hydrogel that transitions from

64 liquid to solid under temperature upshifts. Pluronic solution is maintained at $14^{\circ} \mathrm{C}$ before transfer

65 to the well plate, which allows the solution to stay liquid until it equilibrates to room temperature

66 and gels to trap the nematodes. A chemoattractant is then added to the surface, encouraging the

67 animals to burrow to the surface of the gel. Individuals can be scored based on the time they take

68 to burrow to the surface, while with populations, the number of animals reaching the surface is

69 counted at regular time intervals.

70 The second physical environment we tested is liquid, in which swim motion is assayed using the

71 C. $\underline{\text { elegans }} \underline{\text { Swim }}$ Test (CeleST), analyzed with software that evaluates 8 measures related to

72 body morphology and activity $[35,36]$. The four morphological measures include (i) body wave

73 number - a measure of waviness of the body posture, (ii) asymmetry - the degree to which the

74 animal bends more toward one side or the other, (iii) stretch - a measure of how deep or flat the

75 body bends are, and (iv) curling - percentage of time that an animal spends overlapping with

76 itself. The four activity-related measures include (i) wave initiation rate - the number of body

77 waves initiated by the head or tail per minute, (ii) travel speed - the distance travelled during a

78 defined time, (iii) brush stroke - area painted by the animal body in a complete stroke, and (iv)

79 swim activity index - brush stroke normalized by the time taken to perform two strokes.

80 The third environment features pillars that are deformed while the animal crawls in a

81 microfluidic chamber, enabling calculation of muscle forces [34]. This NemaFlex assay involves 


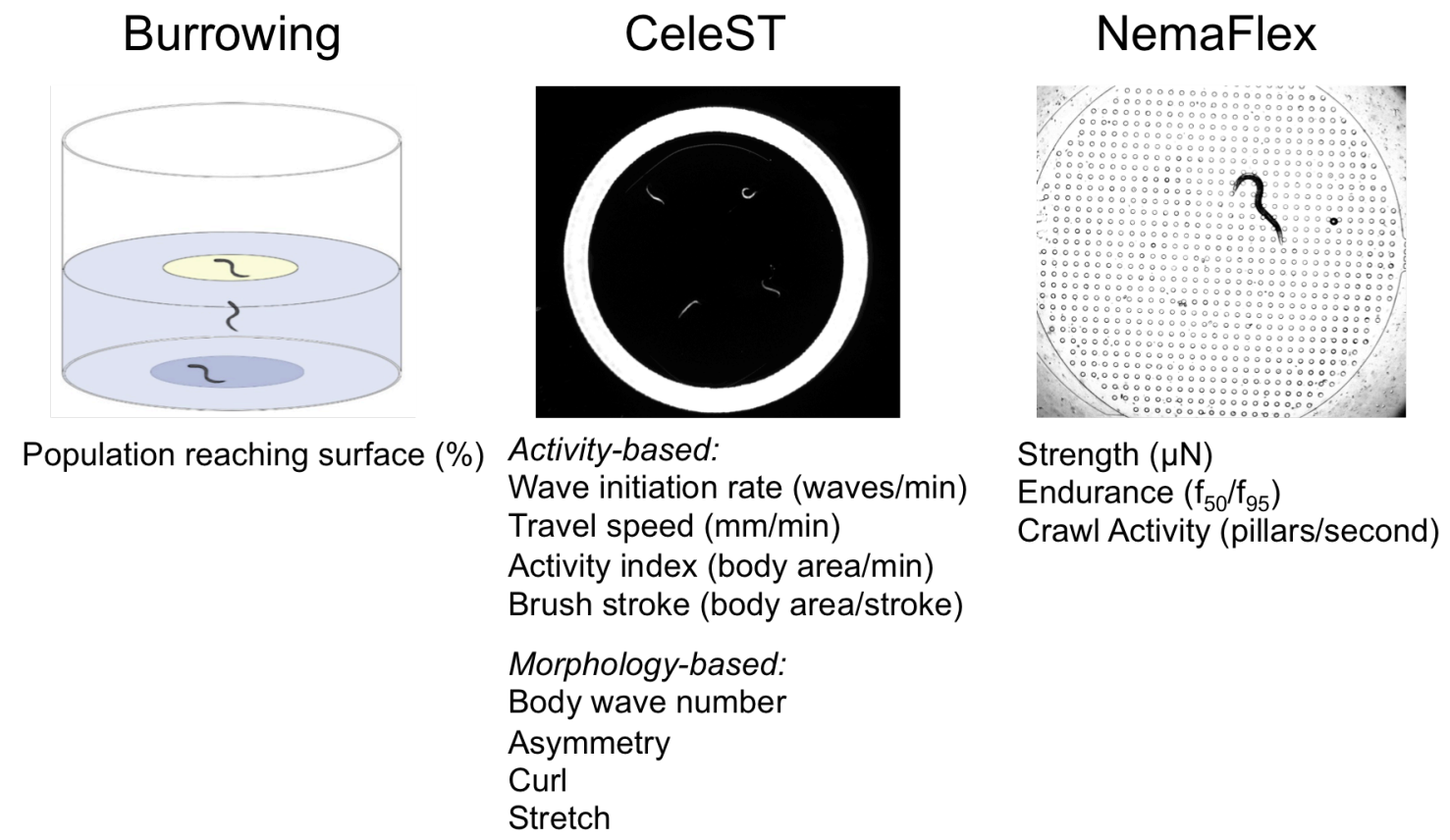

Fig. 1. Overview of the multi-environment phenotyping (MEP) platform for evaluation of the physical performance of C. elegans. The MEP platform consists of a hydrogel-based burrowing assay, the CeleST swim assay, and the NemaFlex assay. From these three platforms, animal populations are assessed for their capacity to burrow in 3D, swim, and crawl, respectively. A total of twelve different measures are extracted from the three distinct mechanical environments.

82

83 imaging in a microfluidic device in which the nematode adopts a crawling gait due to the

presence of a resistive array of flexible micropillars [34, 37]. From these images, pillars with

maximum deflections are extracted, and these deflections are translated to maximal forces.

Muscle strength is defined as the $95^{\text {th }}$ percentile of the maximal forces or $f_{95}$. In addition to measuring the muscle strength from the NemaFlex assay, we also extract measures of muscular endurance and crawling activity. We define muscular endurance as the ratio $f_{50} / f_{95}$, where $f_{50}$ represents $50^{\text {th }}$ percentile of the maximal forces. Animals that exert a high force as a one-time event would have a low ratio while those exerting large forces continuously would have a high ratio. Thus, the ratio $f_{50} / f_{95}$ is taken as a measure of muscular endurance. The crawling activity is calculated from the number of microfluidic pillars that the animal interacts with per unit time. 
94 An individual's physical performance is distinct in different mechanical environments

96 Given that the burrowing, CeleST, and NemaFlex assays expose C. elegans to different

97 mechanical environments, we wondered whether an individual that performs well in one

98 environment would also perform better in a different environment. For example, if an animal

99 shows good swim performance based on CeleST measures, the question is whether the same

100 individual would show improved muscle strength as reported by the NemaFlex assay. Likewise,

101 if an animal is efficient at burrowing and reaching the gel surface quickly, will this individual

102 also display better swim performance? In addition, we sought to determine whether measures of

103 physical performance obtained from different environments correlated with one another.

104 To address how different the physical performance of an individual is in each of our distinct

105 mechanical environments, we phenotyped the same animal using burrowing, CeleST, and

106 NemaFlex assays. We loaded wild-type animals into well plates with the pluronic gel, allowed

107 them to burrow toward the surface, and measured the burrowing time for each individual. As

108 each animal reached the gel surface, we immediately transferred it to a seeded NGM plate. The

109 individuals were allowed to recover for 4 hours to allay any potential burrowing fatigue. We then

110 transferred each individual into a liquid drop briefly for the CeleST assay while maintaining the

111 identity of each animal from the burrowing assay. Finally, each of the indexed individuals was

112 loaded into the NemaFlex device chambers for characterization of crawling and strength

113 measures. 
114 To assess inter- and intra-environment dependence, we determined the Pearson correlation

115 coefficients for each combination of score metrics (Figure 2A). The correlation values between

116 all variables are represented as the intensity value of the heat map. We find some strong

117 correlations among the CeleST measures extracted from the swimming environment.

118 Morphology-related measures of body wave number, asymmetry, and stretch all positively

119 correlate with one another, while activity-based measures of wave initiation rate, travel speed,

120 brush stroke, and swim activity also positively correlate with one another. Variables between the

121 two morphology- and activity-related categories are negatively correlated with one another.

122 Since it has previously been reported that the values of the morphological measures tend to

123 increase with age and the activity-based measures tend to decrease in value with age [35],

124 wild-type aging is at least one context in which these measures are correlated in a similar manner

125 at a population level. Crawling-associated measures within the NemaFlex environment correlate

126 weakly with one another.

127 Importantly, measures from different environments have correlation coefficients hovering around

128 zero - indicating the different environments in fact reveal different and non-overlapping

129 capabilities. Furthermore, principal component analysis (PCA) based on the correlation matrix of

130 all variables shows that each principal component can explain only a small amount of the

131 variance, with the first component accounting for less than $30 \%$ of the variance (Figure $2 \mathrm{~B}$ ). In

132 fact, seven components are required to explain at least $80 \%$ of the variance of the dataset,

133 indicating that no useful reduction of the dimensionality of the dataset is obtained via a PCA.

134 Our data support the idea that the various measures reported from each of the environments

135 reflect distinct indicators of an animal's physiological health. The results also show that an 


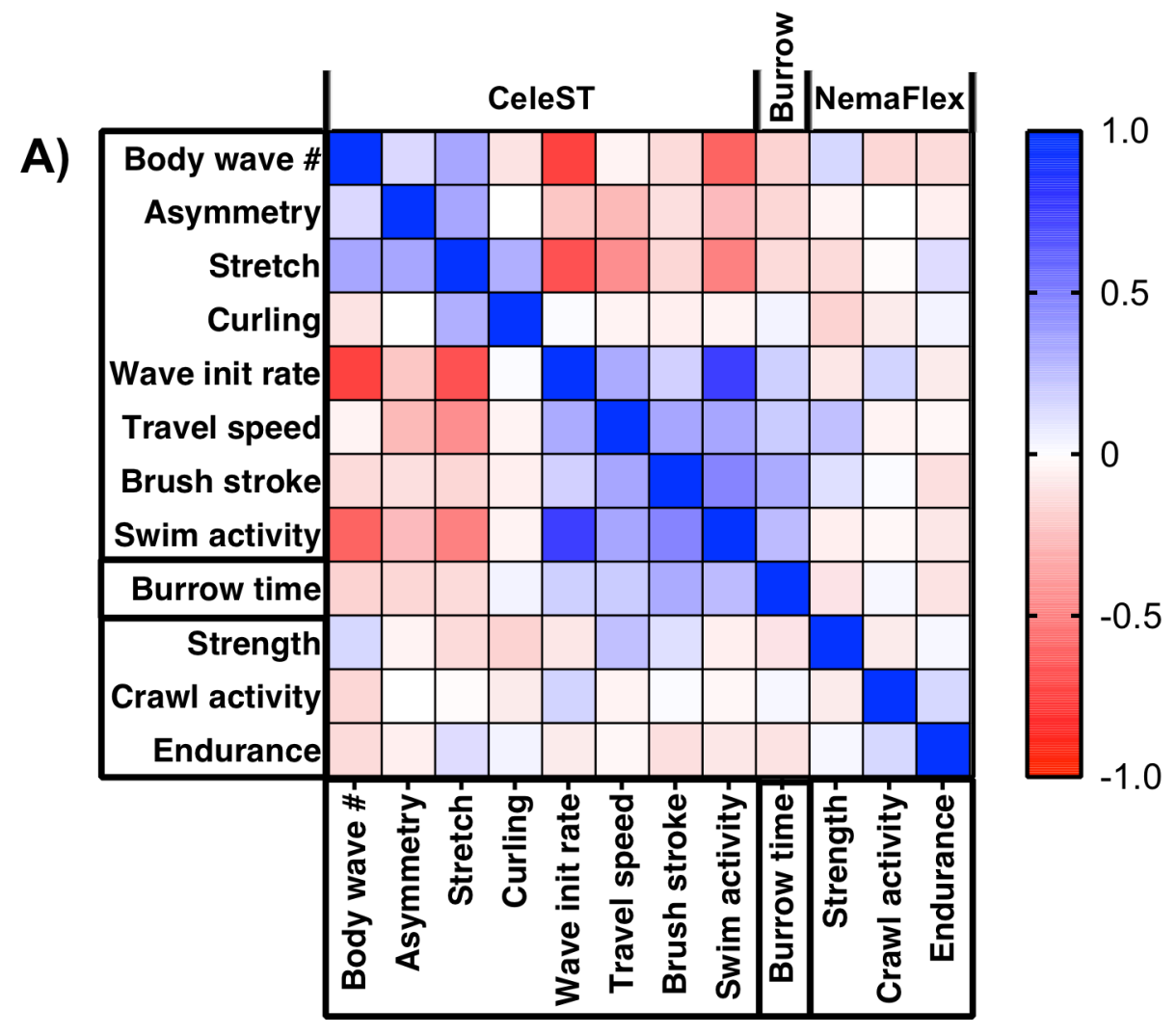

B)

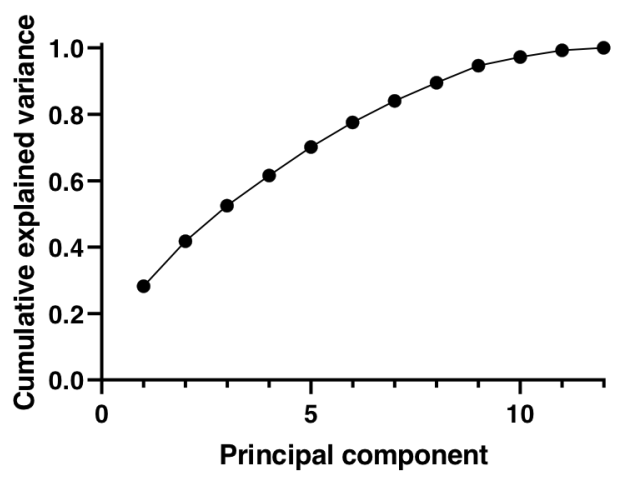

Fig. 2. Measures extracted from the mechanically distinct swimming, crawling, and burrowing environments are not strongly correlated with one another. (A) A heat map shows the correlations among the different parameters extracted from the three different environments used to phenotype the same individual animals. Among all measures, strong correlations exist between only subsets of the CeleST swim measures, and not between the swimming, crawling, and burrowing measures. Intensity values in the heat map show the correlation coefficient obtained from a linear fit of the individual data for each variable plotted against one another. $\mathrm{N}=56$ individual animals tested in all three environments. (B) A principle components analysis based on the correlation matrix does not reduce the dimensionality of the data, as seven components are necessary to explain $80 \%$ of the variation. This means that the data do not form any meaningful smaller sub-dimension in the original 12-dimensional space and therefore report primarily on distinct rather than inter-dependent measures. 
137 individual performing better in one environment may not perform well in a different

138 environment, suggesting that the MEP platform can comprehensively inform on C. elegans

139 physiology.

140 Crawling, swimming, and burrowing environments each elicit a unique transcriptional

141 response

142 Our observations prompted us to ask whether animals exposed to each environment show

143 differences at the gene expression level. We previously reported that placing C. elegans in a

144 swimming environment as compared to a crawling environment elicits a specific gene expression

145 response across a number of different functional clusters of genes, including those affecting

146 oxidative stress response, glucose metabolism, and fat metabolism [38]. Documenting different

147 transcriptional signatures in swimming versus crawling environments would support the value of

148 assaying animals in different environments, as this multi-behavioral test might expose defects or

149 improvements apparent in only a subset of environments.

150 To test for transcriptional profile intersections, we extended our qPCR data that compared

151 changes in gene expression after 90 minutes of swimming to those in a control group of crawling

152 worms [38] by evaluating selected expression in animals after 90 minutes of burrowing relative

153 to a crawling control. Data for both swimming and burrowing animals are shown as the $\log _{2}$ fold

154 change relative to a crawling group that was left on unseeded NGM plates during the 90-minute

155 swim or burrowing period (Figure 3).

156 First, in animals that had burrowed for 90 minutes, we assessed five of the previously reported

157 oxidative stress response genes that respond to swimming (sod-3, sod-5, ctl-2, gst-4, and 


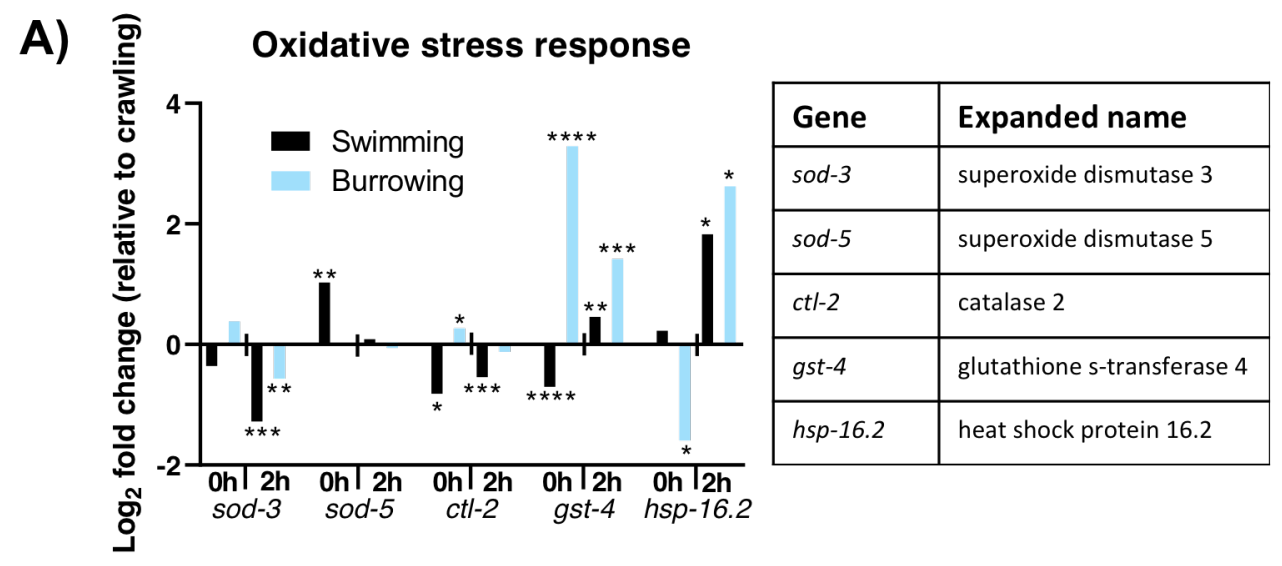

B)

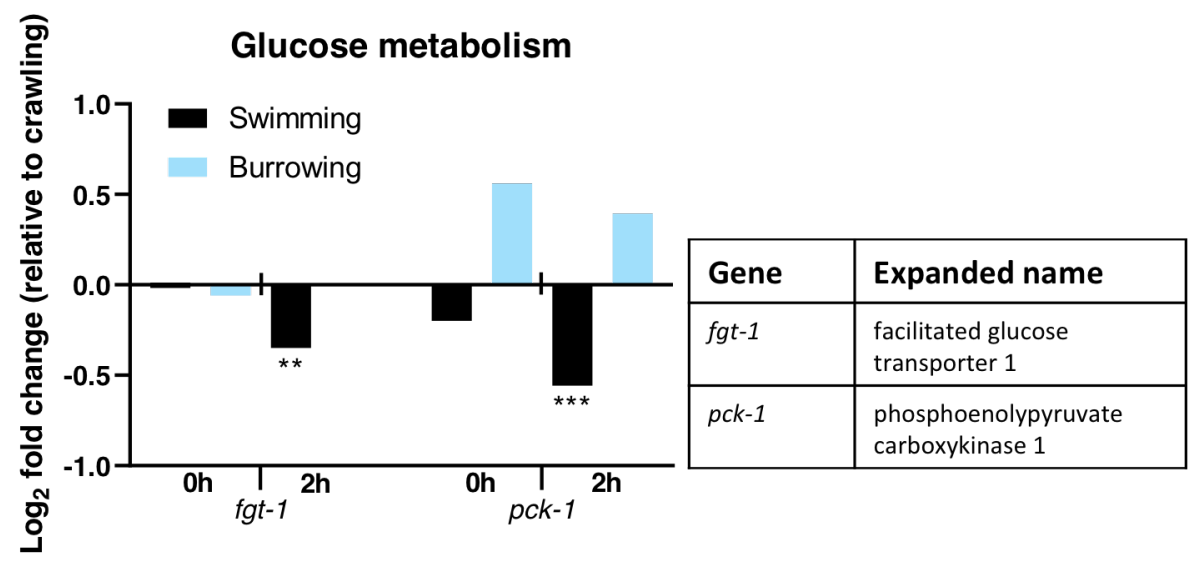

C)

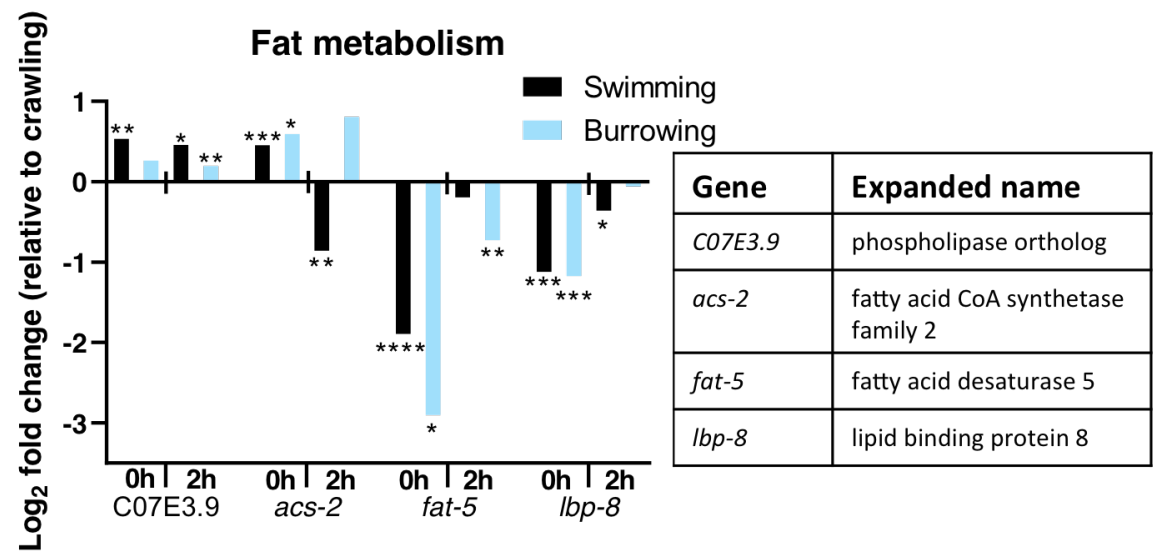

Fig. 3. Swimming, burrowing, and crawling each elicit different gene expression responses. Animals that swim or burrow for 90 minutes differentially express genes involved in (A) oxidative stress response, (B) glucose metabolism, and (C) fat metabolism. Changes in expression are compared to a control group of animals that crawled on unseeded NGM plates for the 90 -minute period. $0 \mathrm{~h}$ is immediately after the 90 -minute period; $2 \mathrm{~h}$ is 2 hours after cessation of the activity trial period. With the permission of the authors, gene expression data from animals after 90 minutes of swimming is reanalyzed from published data in Ref. 38. For each of burrowing and crawling control populations, $\sim 30$ animals were collected, and experiments were done in triplicate. Significance was assessed with a 2-sample t-test. *, $\mathrm{P} \leq 0.05 ; * *, \mathrm{P} \leq 0.01 ; * * *, \mathrm{P} \leq 0.001 ; * * * *, \mathrm{P} \leq 0.0001$. 
$159 h s p-16.2)$ (Figure 3A). Immediately after the burrowing or swimming at the $0 \mathrm{~h}$ time point, there

160 were some notable differences between the swimming and burrowing animals. While the

161 superoxide dismutase 3 (sod-3) gene was not differentially regulated in burrowing vs. swimming

162 animals, sod-5 was significantly upregulated in animals that swam but was not changed in

163 animals that burrowed. Catalase (ctl-2) and $g s t-4$ were significantly upregulated in burrowing

164 animals but downregulated in swimming animals, while $h s p-16.2$ was not changed in animals

165 that swam but was largely downregulated in animals that burrowed. These results indicate that

166 burrowing animals have an immediate transcriptional oxidative stress response distinct from that

167 of crawling or swimming animals.

168 At the 2-hour time point, oxidative stress-related expression was fairly similar between animals

169 that swam or burrowed. The time point 2 hours after challenge cessation may begin to reflect

170 longer term adaptive changes. Notably, 2 hours after swim cessation, animals that swam

171 downregulated the expression of glucose metabolism genes $f g t-1$ and $p c k-1$, which are involved

172 in glucose transport and gluconeogenesis, respectively. Neither fgt- 1 nor $p c k-1$ was

173 downregulated by burrowing (Figure 3B). Finally, the fat metabolism genes assessed here

174 (C07E3.9, acs-2, fat-5, and lbp-8) showed a similar overall pattern in expression changes

175 between burrowing and swimming animals; however, swimming and burrowing animals showed

176 remarkably different expression patterns than their crawling counterparts (Figure 3C).

177 Taken together, analysis of a subset of genes with roles in the oxidative stress response, glucose

178 metabolism, and fat metabolism supports the idea that the environments/experiences of

179 burrowing, swimming, and crawling place distinct demands on animal physiology. This analysis,

180 along with the individual-level physical performance phenotyping data from Figure 2, supports 
181 potential utilization of MEP to more comprehensively assess an animal's physiological health.

A)

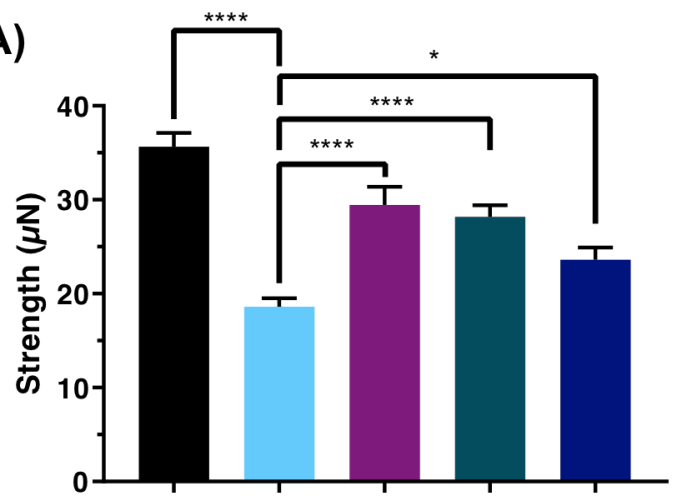

B)

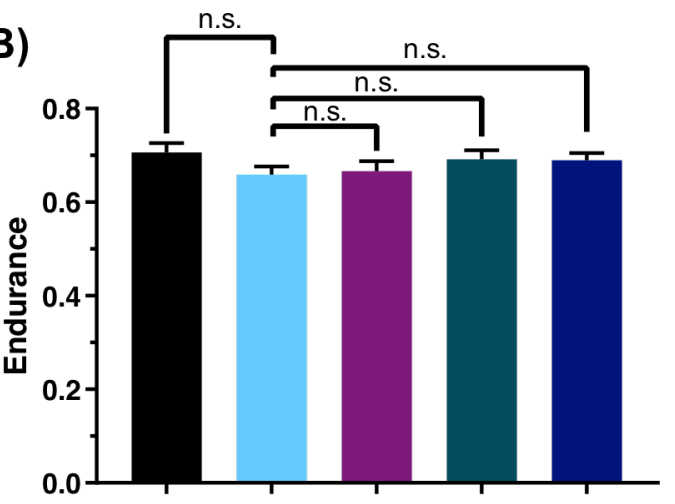

C)

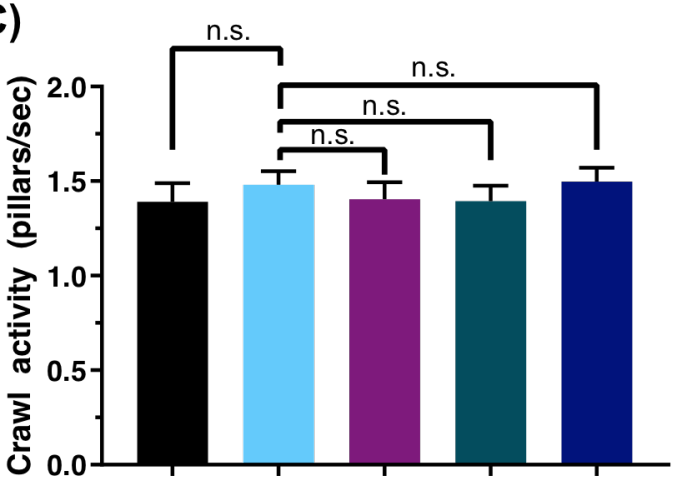

WT

dys-1

dys-1 + prednisone

dys-1 + serotonin

dys-1 + melatonin

Fig. 4. NemaFlex crawling environment: dystrophin mutants are deficient in their strength, which is improved by prednisone, serotonin, and melatonin. (A) We measured strength of animals crawling in a pillar matrix push against pillars in the NemaFlex microfluidics device. dys-1(eg33) mutants are deficient in their strength compared to wild-type animals. All three compounds tested improve animal strength, with prednisone yielding the largest improvement. (B) Endurance of $d y s-1$ (eg33) mutants is not significantly different from that of wild type and also remains unaffected under treatments.

(C) The crawling activity, which is defined as the number of pillars interacted with per second, of dys-1(eg33) is also not different from wild type and does not change under any treatments. WT: $\mathrm{N}=28$; $d y s-1: \mathrm{N}=37 ; d y s-1+$ prednisone: $\mathrm{N}=33 ; d y s-1+$ serotonin: $\mathrm{N}=35 ; d y s-1+$ melatonin: $\mathrm{N}=37$. Significance between WT and $d y s-1$ controls was assessed with a 2-sample t-test; significance between $d y s-1$ and $d y s-1+$ treatments was assessed with one-way ANOVA with Dunnett's post-hoc test. n.s., not significant; *, $\mathrm{P} \leq 0.05 ; * * * *, \mathrm{P} \leq 0.0001$.

We have shown that the MEP platform is suitable for assessing the physical performance of 
186 utility of our approach, we utilized the MEP framework to assess a C. elegans disease model for

187 Duchenne muscular dystrophy (DMD), in which the nematode physical performance is greatly

188 impaired and this decline in physical performance is of clinical relevance. For this purpose, we

189 chose $d y s-1$ (eg33) mutants that are defective in the C. elegans dystrophin gene. We were also

190 curious to know which of the measures of physical performance would be improved when $d y s-1$

191 animals were treated with drugs that have been previously tested in worm and mouse models of

192 DMD. We chose prednisone and melatonin as drug interventions since prednisone is the standard

193 of care for DMD in humans [39], and melatonin has been tested in humans with some beneficial

194 effects on disease pathology [40]. We also included serotonin, as serotonin was shown to be

195 capable of preventing muscle damage in a C. elegans DMD model [41], although serotonin alone

196 was not found efficacious in a follow-up study in a mouse DMD model [42].

197 Crawling environment in NemaFlex devices. Using NemaFlex, we previously reported that

$198 d y s-1($ eg33) mutants are significantly weaker than their wild-type counterparts on the third day

199 of adulthood [37]. Here, using a microfluidic device optimized for younger adult animals, we

200 show that $d y s-1$ mutants are significantly weaker than wild-type animals on the second day of

201 adulthood (Figure 4A). The ability to detect this weakness at an early time point is relevant in

202 that patients with DMD exhibit muscle weakness early in life and intervention testing in the

203 worm model can now be focused on earlier outcomes.

204 With an interest in measuring the efficacy of clinical interventions aimed at alleviating the $d y s-1$

205 pathophysiology, we found that treating $d y s-1$ mutants with prednisone and melatonin induces

206 significant improvements in muscle strength over the control (Figure 4A). Serotonin, which was

207 previously reported to reduce the number of damaged muscle cells in the sensitized $d y s-1 ; h l h-1$ 

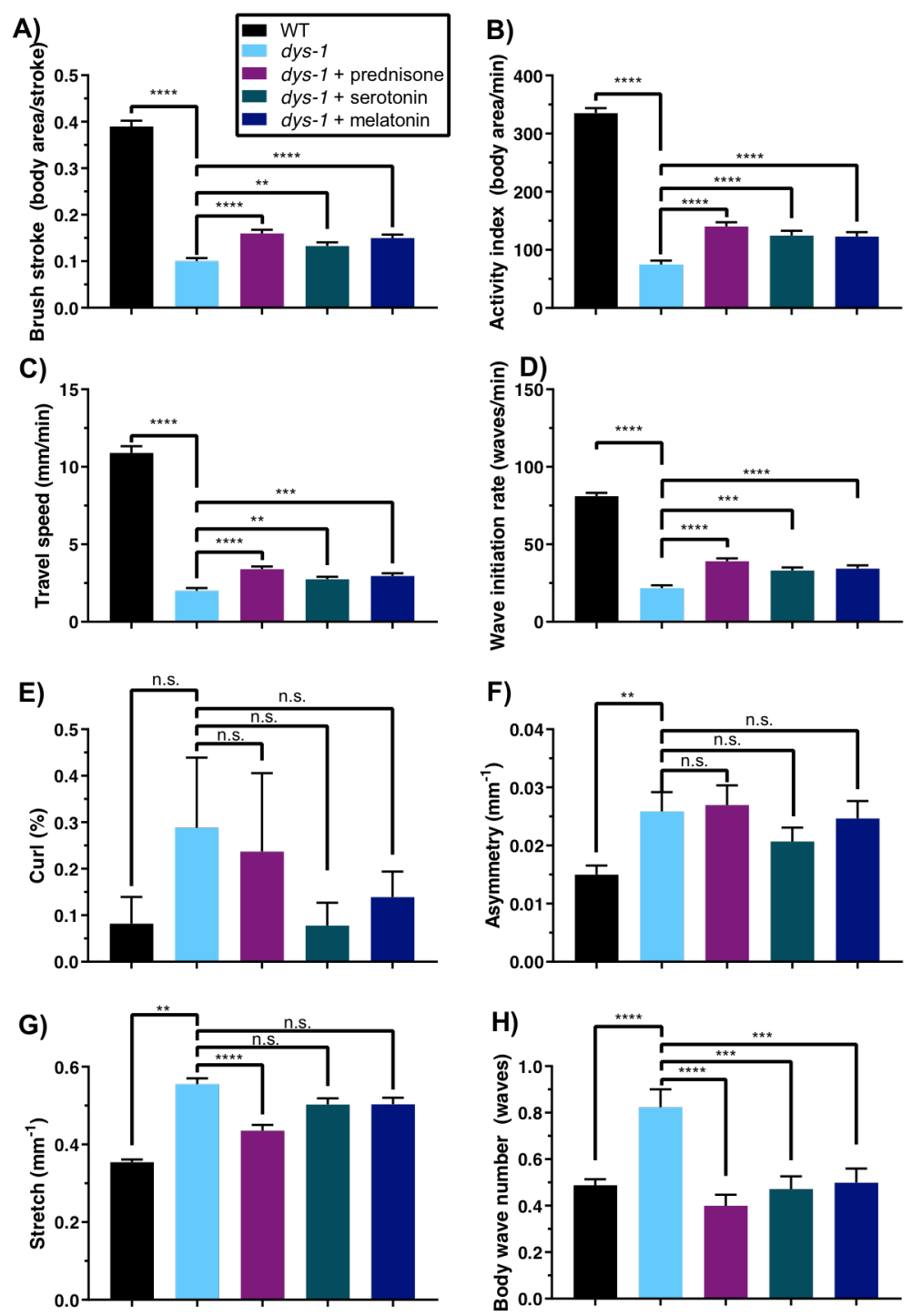

Fig. 5. Swimming environment: $d y s-1$ (eg33) mutants are deficient in nearly all swimming measures, and treatments with prednisone, serotonin, and melatonin improve most measures. dys-1(eg33) mutants have significantly lower (A) brush stroke, (B) activity index, (C) travel speed, and (D) wave initiation rate compared to wild type. Treatments with prednisone, serotonin, and melatonin give significant improvements in these measures. (E) dys-1 (eg33) mutants are not significantly different from wild type in their curling percentage, but their (F) asymmetry, $(\mathrm{G})$ stretch, and $(\mathrm{H})$ body wave number are significantly higher than those of wild type, and all compounds improve these measures, with the exception of asymmetry, which is not improved under any treatment, and stretch, which is improved only by prednisone. All four of these measures, which reflect body posture, increase with age in wild-type animals. WT: $\mathrm{N}=56 ; d y s-1: \mathrm{N}=56$; $d y s-1+$ prednisone: $\mathrm{N}=55$; $d y s-1+$ serotonin: $\mathrm{N}=54$; $d y s-1+$ melatonin: $\mathrm{N}=56$. Significance between $\mathrm{WT}$ and $d y s-1$ controls was assessed with a 2-sample t-test; significance between $d y s-1$ and $d y s-1+$ treatments was assessed with one-way ANOVA with Dunnett's post-hoc test. n.s., not significant; **, $\mathrm{P} \leq 0.01$; ***, $\mathrm{P} \leq 0.001$; ****, $\mathrm{P} \leq 0.0001$. 
model [41], also improves muscle strength of the $d y s-1$ mutant. While all three compounds

210 improve strength of the $d y s-1$ mutant, none are able to restore strength back to wild-type levels in

211 the strength assay, indicating these treatments do not fully reverse the muscular defects of the

$212 d y s-1$ mutant. In addition, we find that measures of muscle endurance and crawling activity are

213 not significantly different in the $d y s-1$ mutant and also do not change under treatment with any of

214 the compounds (Figure 4B-C). Thus, treatments that confer positive effects in humans also

215 confer positive effects in C. elegans, but differences are not revealed in all the measures from

216 NemaFlex assay.

217 CeleST swimming environment. $d y s-1$ mutants are deficient in their thrash rate in liquid, a

218 single-parameter readout of swimming ability [37]. We were therefore interested in assessing

219 whether the various activity- and morphology-related measures of CeleST were also deficient in

220 swimming $d y s-1$ animals. We found a striking difference between $d y s-1$ mutants and wild-type

221 animals in all four activity-related measures, such that brush stroke, activity index, travel speed,

222 and wave initiation rate are each a small fraction of baseline values for $d y s-1$ as compared with

223 control animals (Figure 5A-D). Prednisone, serotonin, and melatonin, each of which improves

224 muscle strength of $d y s-1$ mutants, also confer modest but significant improvements in each of

225 these four activity-related swim measures. Additionally, while $d y s-1$ mutants do not appear to

226 spend more time in a curled morphology compared with wild-type animals (Figure 5E), they do

227 have significantly higher asymmetry, stretch, and body wave number (Figure 5F-H). Prednisone,

228 serotonin, and melatonin are able to restore body wave number closer to wild-type levels but

229 have no significant effect on the abnormally high asymmetry of $d y s-1$ animals. Only prednisone

230 is able to restore abnormally high stretch closer to wild-type levels; serotonin and melatonin have 
231 no significant effect. These results in the swim environment partially mirror the result of

232 pharmacological treatments in improving strength: although the compounds do have

233 advantageous effects on deficiencies, treated $d y s-1$ animals still trail behind wild-type animals.

234 Furthermore, the resistance of animal asymmetry to the various treatments and the resistance of

235 stretch to two of the three treatments indicate that these compounds are not targeting all aspects

236 of physiological abnormalities in the $d y s-1$ mutants.

237 Burrowing environment. dys-1(eg33) mutants exhibit burrowing defects [31, 43], which we

238 sought to confirm using our novel hydrogel-based burrowing platform. Here, we assessed the

239 burrowing ability of $d y s-1$ animals that had been treated with pharmacological interventions,

240 which is the first report of how pharmacological interventions impact burrowing ability in $C$.

241 elegans. We find that while over $80 \%$ of wild-type animals reach the surface by the end of the

242 2-hour burrowing assay, only about 5\% of $d y s-1$ mutants reached the surface during this same

243 time frame (Figure 6). The strong defect in $d y s-1$ burrowing ability suggests that burrowing may

244 place a high demand on the muscle and requires intact excitation-contraction coupling in the

245 muscle. These defects are only partially addressed by prednisone, melatonin, and serotonin, as

246 each compound gives significant but modest improvements in the burrowing ability of $d y s-1$,

247 although treated animals still fall short of wild-type performance (Figure 6). Our data support the

248 notion that the burrowing assay enhances the dynamic range in which we can evaluate drug

249 interventions that counter $d y s-1$ deficits. 


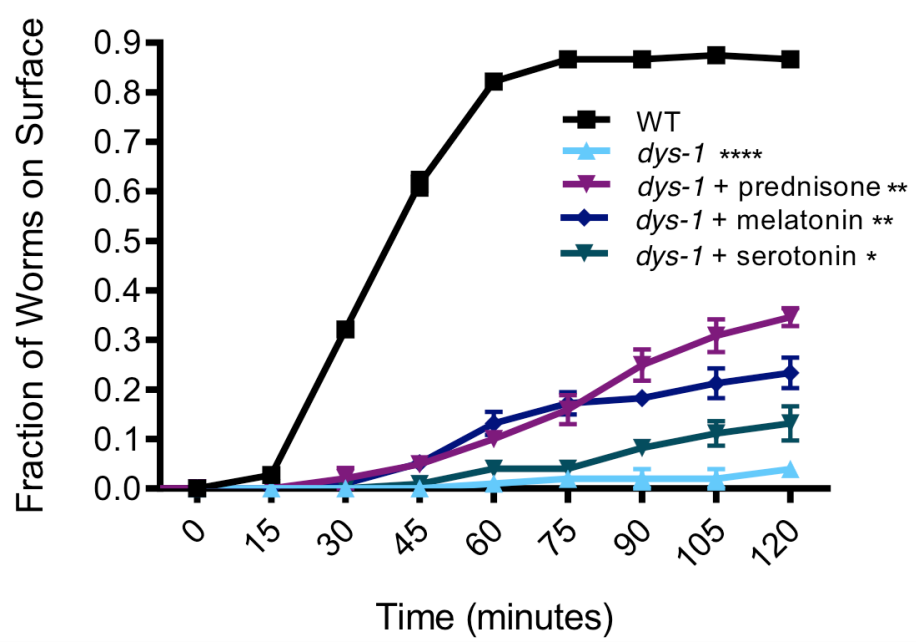

Fig. 6. Burrowing environment: dystrophin mutants are highly deficient in burrowing and some interventions modestly improve this deficiency. dys-1(eg33) mutants are highly deficient in burrowing, with only less than $10 \%$ of the population able to reach the surface compared to over $\sim 80 \%$ of wild-type animals after a 2 hour trial. Prednisone, melatonin, and serotonin confer a significant improvement in the burrowing ability of $d y s-1$ (eg33) mutants, although their performance still falls short of that of wild type. Trials were in triplicate with average samples sizes of: WT: $\mathrm{N}=37$; dys-1: $\mathrm{N}=34$; dys-1+prednisone: $\mathrm{N}=34$; dys-1+serotonin: $\mathrm{N}=33$; dys-1+melatonin: $\mathrm{N}=33$. Significance was assessed with two-way ANOVA. n.s., not significant; *, $\mathrm{P} \leq 0.05 ; * *, \mathrm{P} \leq 0.01 ; * * * *, \mathrm{P} \leq 0.0001$.

\section{DISCUSSION}

\section{A novel multi-environment phenotyping platform}

253 Assessment of physical performance in humans is not only central to a variety of disease

254 contexts but is also critical for evaluating the effects of factors such as aging, diet, and exercise.

255 Development of measures that are indicative of physical performance in C. elegans that are

256 analogous to human measures is expected to increase the translational relevance of this genetic

257 model. Behavioral assays that extract descriptive measures of C. elegans swim locomotion [26,

$25835,36]$, crawling on NGM plates [22, 27, 44-46], and burrowing [24, 31] have been documented,

259 but none have investigated individuals in these different environments in the context of assessing 
260 physical performance. We suggest that our approach that integrates multiple measures from three

261 unique physical environments advances the general field of phenotyping in C. elegans.

262 In this study, we establish the value of this MEP platform by showing that the parameters

263 reported are not redundant with one another - testing in multiple environments increases the

264 "bandwidth" over which deficits can be detected and improvements can be documented. Our

265 gene expression data show that swimming, crawling, and burrowing place unique demands on

266 the nematode, as evidenced by analysis of a sample set of genes representative of oxidative stress

267 response, glucose metabolism, and fat metabolism, which are differentially expressed depending

268 on the animal's environment and specific locomotory response. The environments of swimming,

269 crawling, and burrowing can put different demands on the organism (e.g. oxygen, pressure, and

270 physical forces) and its physiology (muscle demands and type of movement).

\section{Application of MEP to C. elegans DMD model}

272 Previous work reports studies of $d y s-1$ mutant deficits using single parameters such as crawling

273 speeds [47], swimming speeds [48], burrowing ability [31], or muscle strength [37]. Additional

274 work that did give multiple readouts of $d y s-1$ or $d y s-1, h l h-1$ animal physiology established

275 baseline values for a few additional parameters within the swimming environment using the

276 Biomechanical Profiling (BMP) platform but did not look at the effects of any interventions [26,

277 49]. More recently, one study evaluated $d y s-1$ mutants in swimming, crawling, and burrowing

278 environments and focused solely on the frequencies and amplitudes of the animal motion in the

279 different environments [43]. 
280 Building on this prior work, we applied the MEP platform to test whether additional deficiencies

281 could be detected in the same animal populations when multiple phenotypic measures were

282 assayed. While crawling activity, muscular endurance, and curl percentage are not significantly

283 different in $d y s-1$ mutants as compared with their wild-type counterparts, all other nine measures

284 we report are strikingly worse. These nine measures span each of the three physical

285 environments, indicating that we can detect deficiencies in the same animal population in all

286 environments comprising the MEP platform. To establish the efficacy of the platform in

287 detecting improvements from pharmacological interventions, we assessed animals that had been

288 treated with prednisone and melatonin, previously reported to improve muscle strength [37], as

289 well as serotonin, which had been previously reported to reduce the number of damaged muscle

290 cells in the dys-1; hlh-1 DMD model [41, 50].

291 The findings from our study here inform two potential directions in researching therapeutic

292 compounds for DMD. First, any compounds that counter defects in all the measures reported in

293 the MEP platform would be most promising to pursue in $m d x$ mice and eventually clinical trials.

294 Second, compounds that restore a subset of the deficiencies to wild-type levels may be worth

295 pursuing and might be easily optimized in the facile $C$. elegans model. Similar to our study with

296 the DMD model, future opportunities also exist to test other C. elegans disease models in which

297 the neuromuscular system is impaired to potentially identify candidates for therapeutic

298 intervention. 


\section{CONCLUSIONS}

300 In this work, we have shown the power of a multi-environmental phenotyping platform that

301 holds potential for improving translation of findings in C. elegans disease models to humans.

302 The three different physical environments, which elicit distinct locomotory modes of crawling,

303 swimming, and burrowing, each also challenge the animal in distinct manners, as demonstrated

304 by the unique information extracted from each environment and the differences in expression of

305 select genes in each environment. We demonstrated the utility of this framework by assessing the

306 health of the C. elegans model for Duchenne muscular dystrophy, both with and without

307 pharmacological interventions. The framework we describe here can be utilized in the future for

308 robustly assessing health and determining interventions that might be most successfully

309 translated from C. elegans disease models to mammalian systems.

\section{MATERIALS AND METHODS}

\section{Nematode strains, culture, and drug treatments}

313 Strains used in this experiment were wild-type (N2 strain) and $d y s-1$ (eg33) (BZ33 strain). The

314 strains were provided by the Caenorhabditis Genetics Center (CGC). For all drug treatments,

315 stock solutions were aliquoted on the surface of seeded NGM plates approximately 24 hours

316 before using the plates. The final concentration of the drug was calculated based on the total

317 volume of the contents of the plate. Concentrations used for drug treatments were matched with

318 concentrations used in previous studies: $0.37 \mathrm{mM}$ prednisone [37], $1 \mathrm{mM}$ melatonin [37], 
$2.5 \mathrm{mM}$ serotonin creatinine sulfate [41]. A control solvent was added to the surface of agar plates used for culturing animals that did not receive a drug treatment.

321 Synchronized C. elegans populations were prepared by picking 20-30 adult animals to each

322 seeded plate and allowing them to lay eggs for $\sim 3-4$ hours. At the end of this time period, adult

323 animals were picked and discarded, leaving synchronized eggs on the plate. Eggs were allowed

324 to hatch and grow at $20^{\circ} \mathrm{C}$ until the first or second day of adulthood, when all assays were

325 conducted. Animals for all assays were Day 2 adults, except for animals used for gene

326 expression and burrowing experiments, which were Day 1 adults. For assays done on Day 2 of

327 adulthood, animals were transferred on Day 1 to fresh plates in order to ensure sufficient food

328 and minimal crowding.

\section{NemaFlex strength assay}

330 The NemaFlex strength assay was conducted as previously described, with a few modifications

$331[34,37]$. In our previous study, we used a microfluidic device design that was optimized to

332 accommodate animals of a much wider age range [37]. For the present study, we instead utilized

333 a device with modified pillar diameter and spacing that is best used for Day 1 and Day 2 adults.

334 Here, the pillars had a smaller diameter $(\sim 41 \mu \mathrm{m})$ and tighter spacing $(74 \mu \mathrm{m}$ between edges of

335 the pillars) due to the smaller size of the worms at the beginning of adulthood. Because of this,

336 the force constants calculated from this device are not as large as those calculated using the

337 device in our previous study [37]; this therefore results in smaller force values. However, the

338 same device is used for all experiments within this study, which allows for consistency in the

339 comparisons of the data presented here. 
340 Synchronized animals were loaded into the devices with one animal per chamber. A 60-second

341 video was collected for each animal using a Nikon TI-E microscope with a 4x objective and

342 Andor Zyla sCMOS 5.5 camera at a frame rate of 5 FPS. Movies were then processed and

343 analyzed for force values using our in-house-built image processing software (MATLAB,

$344 \mathrm{R} 2015 \mathrm{~b}$ ). Animal strength is determined by selecting for the maximum force exerted in each

345 frame (300 total frames and therefore force values per animal), and then selecting for the $95^{\text {th }}$

346 percentile of these forces. The $95^{\text {th }}$ percentile is selected rather than the absolute maximum, as it

347 is more robust to error. Two new measures presented here are endurance and crawling activity.

348 Endurance is calculated in a similar way to strength, but instead the $50^{\text {th }}$ percentile of the

349 maximum forces exerted is normalized by the strength, or the $95^{\text {th }}$ percentile. Thus, this is the

350 average force exerted normalized by our definition of strength and gives an idea of the profile of

351 forces being exerted by the animal. Crawling activity is defined as the number of pillars that the

352 animal interacts with per second, where pillars are automatically counted by image processing

353 software, and the count of the pillars only needs to be normalized to the duration of the acquired

354 movie. Significances between wild type and $d y s-1$ controls were assessed using a 2 -sample $t$-test.

355 Significance between $d y s-1$ and $d y s-1+$ treatments was assessed with one-way ANOVA with

356 Dunnett's post-hoc test.

\section{CeleST swim assay}

358 C. elegans $\underline{\text { Swim }} \underline{\text { Test }}$ (CeleST) assays were conducted as previously described $[35,36]$. Animals

359 were picked and placed into a $50 \mu \mathrm{L}$ aliquot of M9 Buffer on a specialized glass slide with two,

$360 \quad 10-\mathrm{mm}$ pre-printed rings on the surface (Thermo Fisher Scientific), as previously used by the

361 original developers of the CeleST assay. The rings hold in the M9 buffer and act as a swim arena 
362 for the animals. Four to five animals were placed into each M9 buffer aliquot, and two swim

363 arenas were loaded at a time and then imaged sequentially. Images were acquired with a Nikon

364 TI-E microscope and Andor Zyla sCMOS 5.5 camera at a frame rate of $\sim 12$ FPS. Images were

365 acquired using dark-field imaging to allow a bright worm on a dark background. Images were

366 processed using the previously developed CeleST software, although the most recent version of

367 the software includes 8 of the originally reported 10 measures; the measures of reverse

368 swimming and attenuation have been dropped from recent releases of the CeleST software and

369 were thus not considered for the experiments described here. The CeleST software reports the

370 percentage of frames where a worm was successfully and accurately segmented, and only these

371 valid frames are used towards the calculation of all measures. An approximate validity cutoff of

$37280 \%$ was implemented to ensure that sufficient and continuous frames were available for

373 measure computation. Significances between wild type and $d y s-1$ controls were assessed using a

3742 -sample $t$-test. Significance between $d y s-1$ and $d y s-1+$ treatments was assessed with one-way

375 ANOVA with Dunnett's post-hoc test.

\section{Pluronic gel-based burrowing assay}

377 Burrowing assays were conducted as previously described [25]. Briefly, approximately 30 to

37840 C. elegans were introduced into the base of a well of a 12-well plate by handpicking with a

379 platinum wire into a small pluronic drop. Transfer of $E$. coli to the well plate was avoided by first

380 transferring animals to an unseeded NGM plate and letting them crawl for a few minutes before

381 the animals were added to the well plate. A volume of $2.5 \mathrm{~mL}$ of pluronic was added to form the

382 upper layer of the gel, which gave a gel height of about $0.75 \mathrm{~cm}$. The exact number of animals

383 loaded into the well was then counted and recorded. Once the gel had solidified, $20 \mu \mathrm{L}$ of 
concentrated $E$. coli solution was added to the surface above the animals. The concentrated

385 E. coli solution was made by re-suspending an E. coli pellet in liquid NGM, which is just NGM

386 without the agar. The addition of the $E$. coli solution marked the $t=0$ time point of the assay.

387 The number of animals on the surface was then scored every 15 minutes for a total of 2 hours

388 unless otherwise noted. The percent of the population reaching the surface at each time point was

389 then calculated. For all experiments conducted, a concentration of $26 \mathrm{w} / \mathrm{w} \%$ of Pluronic F-127

390 (Sigma-Aldrich) in DI water was used. Each condition/strain was run in triplicate, and

391 significances were assessed using 2-way ANOVA in GraphPad Prism software.

\section{Individual-level, multi-environment phenotyping}

393 NemaFlex, CeleST, and burrowing assays were conducted as previously described but with a

394 few modifications that enabled sustained tracking of the individual animal identity, which

395 allowed looking at each individual animal under burrowing, crawling, and swimming

396 environments. Animals were loaded into the 12-well plates as described, and the addition of

397 bacteria marked the start of the assay. Rather than counting the number of animals on the

398 surface, however, individual animals were collected from the burrowing wells as they reached

399 the surface. Each animal was given a score of how many minutes it took to reach the surface.

400 Individual animals were placed in a 35mm NGM plate with E. coli OP50 and allowed to recover

401 for 4 hours. Individual animals were then imaged swimming for CeleST analysis (swimming

402 only for a few minutes), and then were placed back on their agar plate. After all imaging was

403 finished for swimming animals, individual worms were loaded into NemaFlex chambers and

404 imaged and analyzed for strength, activity, and muscular endurance as previously described. The

405 only difference in data representation is for that of burrowing ability, where individual animals 
406 were given the score of number of minutes taken to reach the surface. This is in contrast to the

407 typical representation of the percent of the population that has reached the surface at a given time

408 point that is reported when populations rather than individuals are assessed.

\section{Gene expression via quantitative PCR}

410 To assess how C. elegans respond to the three different environments of crawling, burrowing,

411 and swimming, we conducted an assay where wild-type $C$. elegans were allowed to burrow for

41290 minutes, and then we assessed the genetic response as related to oxidative stress, glucose

413 metabolism, and fat metabolism. Animals were loaded into burrowing wells as previously

414 described, except a tall gel layer that filled the well completely to the top was added to ensure

415 that animals did not exit the burrowing environment during the 90 minutes of burrowing. Liquid

416 NGM was added to the surface to provide chemotactic stimulation to the animals without

417 introducing a food source (E. coli). A control group of animals were transferred to unseeded

418 NGM plates for the 90-minute duration of the burrowing. At the conclusion of the 90 minutes,

419 approximately 30 each of burrowing and control animals were collected in M9 Buffer. Another

420 set of burrowing and control animals were transferred to seeded NGM plates for recovery, and

421 then were collected after 2 hours.

422 The RNA extraction and qPCR protocols were consistent with those previously described [38,

423 51]. Immediately after collection in M9 Buffer, animals were spun down and the supernatant was

424 removed. After the addition of TRIzol (Ambion), animals underwent snap freezing with liquid

425 nitrogen. The samples then underwent freeze/thaw cycles with liquid nitrogen and a heat block at

$42637^{\circ} \mathrm{C}$. Subsequently, total RNA was extracted as instructed by the manufacturer's protocol 
427 (Ambion), and cDNA was made with the SuperScript III First-Strand Synthesis System

428 (Invitrogen). The PerfeCTa SYBR Green FastMix (Quantabio), $0.5 \mu \mathrm{M}$ of primers for each gene,

429 and diluted cDNA were used for conducting qPCR. The primers used match those previously

430 published as part of the prior analysis done for gene expression changes after 90 minutes of

431 swimming [38]. A 7500 Fast Real-Time PCR System (Applied Biosystems) was used with the

$432 \Delta \Delta \mathrm{Ct}$ method relative expression method for calculations [52]. For reference genes, $c d c-42$ and

433 Y45F10D.4 were used [53].

434 Statistical analyses. All significances were assessed with a 2-sample t-test, except for

435 population burrowing comparisons, which were assessed using two-way ANOVA in GraphPad

436 Prism software, and NemaFlex and CeleST measures for $d y s-1$ vs. $d y s-1+$ treatments, which were

437 assessed with one-way ANOVA with Dunnett's post-hoc test.

\section{ACKNOWLEDGEMENTS}

439 Some strains were provided by the CGC, which is funded by NIH Office of Research

440 Infrastructure Programs (P40 OD010440). We would like to acknowledge Leila Lesanpezeshki

441 for useful discussions surrounding burrowing experiments and for fabricating the mold used to

442 make NemaFlex microfluidic devices. This work is partially supported by funding from the

443 National Institutes of Health (RO1 AG051995-04 to M.D. \& S.V.), National Aeronautics and

444 Space Administration (NNX15AL16G to S.V.), and the Biotechnology and Biological Sciences

445 Research Council (BB/N015894/1 to N.J.S.). J.E.H. acknowledges funding support from the

446 Fulbright U.S. Student Program and the Germanistic Society of America. R.L. has been funded

447 by postdoctoral fellowships from Life Sciences Research Foundation (sponsored by Simons 
bioRxiv preprint doi: https://doi.org/10.1101/2020.08.17.253583; this version posted August 17, 2020. The copyright holder for this preprint (which was not certified by peer review) is the author/funder, who has granted bioRxiv a license to display the preprint in perpetuity. It is made available under aCC-BY 4.0 International license.

448 Foundation; award \# Laranjeiro-2015) and American Heart Association (award

449 \# 18POST33960502). A.A. was supported by the Max Planck Society. 


\section{REFERENCES}

451 1. Caspersen CJ, Powell KE, Christenson GM. Physical activity, exercise, and physical 452 fitness: definitions and distinctions for health-related research. Public health rep.

453 1985;100(2):126-31.

454 2. McDonald CM. Physical activity, health impairments, and disability in neuromuscular 455 disease. American journal of physical medicine \& rehabilitation. 2002;81(11):S108-S20.

456 3. Beaudart C, Rolland Y, Cruz-Jentoft AJ, Bauer JM, Sieber C, Cooper C, et al.

457 Assessment of muscle function and physical performance in daily clinical practice. Calcified 458 Tissue International. 2019:1-14.

459 4. Wang L, Larson EB, Bowen JD, van Belle G. Performance-based physical function and 460 future dementia in older people. Archives of internal medicine. 2006;166(10):1115-20.

461 5. Guralnik JM, Simonsick EM, Ferrucci L, Glynn RJ, Berkman LF, Blazer DG, et al. A 462 short physical performance battery assessing lower extremity function: association with self463 reported disability and prediction of mortality and nursing home admission. Journal of 464 gerontology. 1994;49(2):M85-M94.

465 6. Studenski S, Perera S, Wallace D, Chandler JM, Duncan PW, Rooney E, et al. Physical 466 performance measures in the clinical setting. Journal of the American Geriatrics Society. $467 \quad 2003 ; 51(3): 314-22$.

468 7. Volpato S, Cavalieri M, Sioulis F, Guerra G, Maraldi C, Zuliani G, et al. Predictive value 469 of the Short Physical Performance Battery following hospitalization in older patients. The

$471 \quad 8 . \quad$ Rockwood K, Song X, MacKnight C, Bergman H, Hogan DB, McDowell I, et al. A 472 global clinical measure of fitness and frailty in elderly people. Canadian Medical Association 473 Journal. 2005;173(5):489-95.

4749 9. Brenner S. The genetics of Caenorhabditis elegans. Genetics. 1974;77(1):71-94.

475 10. Collins CA, Morgan JE. Duchenne's muscular dystrophy: animal models used to 476 investigate pathogenesis and develop therapeutic strategies. International Journal of 477 Experimental Pathology. 2003;84(4):165-72.

478 11. Nass R, Miller DM, Blakely RD. C. elegans: a novel pharmacogenetic model to study 479 Parkinson's disease. Parkinsonism \& Related Disorders. 2001;7(3):185-91.

480 12. Harrington AJ, Hamamichi S, Caldwell GA, Caldwell KA. C. elegans as a model 481 organism to investigate molecular pathways involved with Parkinson's disease. Developmental 482 Dynamics. 2010;239(5):1282-95. 
13. Link CD. C. elegans models of age-associated neurodegenerative diseases: lessons from transgenic worm models of Alzheimer's disease. Experimental Gerontology. 2006;41(10):100713.

14. Parker JA, Connolly JB, Wellington C, Hayden M, Dausset J, Neri C. Expanded polyglutamines in Caenorhabditis elegans cause axonal abnormalities and severe dysfunction of PLM mechanosensory neurons without cell death. Proceedings of the National Academy of Sciences. 2001;98(23):13318-23.

490 15. Poulin G, Nandakumar R, Ahringer J. Genome-wide RNAi screens in Caenorhabditis 491 elegans: impact on cancer research. Oncogene. 2004;23(51):8340-5.

492 16. Herndon LA, Schmeissner PJ, Dudaronek JM, Brown PA, Listner KM, Sakano Y, et al. 493 Stochastic and genetic factors influence tissue-specific decline in ageing C. elegans. Nature. 494 2002;419(6909):808-14.

495 17. Bushby K, Finkel R, Birnkrant DJ, Case LE, Clemens PR, Cripe L, et al. Diagnosis and 496 management of Duchenne muscular dystrophy, part 1: diagnosis, and pharmacological and 497 psychosocial management. The Lancet Neurology. 2010;9(1):77-93.

498 18. Folch J, Petrov D, Ettcheto M, Abad S, Sánchez-López E, García ML, et al. Current research therapeutic strategies for Alzheimer's disease treatment. Neural Plasticity. 2016;2016.

500 19. Dehay B, Bourdenx M, Gorry P, Przedborski S, Vila M, Hunot S, et al. Targeting $\alpha-$ 501 synuclein for treatment of Parkinson's disease: mechanistic and therapeutic considerations. The 502 Lancet Neurology. 2015;14(8):855-66.

503 20. Ross CA, Tabrizi SJ. Huntington's disease: from molecular pathogenesis to clinical 504 treatment. The Lancet Neurology. 2011;10(1):83-98.

505 21. Kwak JY, Kwon K-S. Pharmacological Interventions for Treatment of Sarcopenia: 506 Current Status of Drug Development for Sarcopenia. Journal of the Korean Geriatrics Society. 5072019.

508 22. Nussbaum-Krammer CI, Neto MF, Brielmann RM, Pedersen JS, Morimoto RI. 509 Investigating the spreading and toxicity of prion-like proteins using the metazoan model 510 organism C. elegans. JoVE (Journal of Visualized Experiments). 2015;(95):e52321.

511 23. Buckingham SD, Sattelle DB. Fast, automated measurement of nematode swimming 512 (thrashing) without morphometry. Bmc Neuroscience. 2009;10(1):1.

513 24. Bainbridge C, Schuler A, Vidal-Gadea AG. Method for the assessment of neuromuscular 514 integrity and burrowing choice in vermiform animals. Journal of Neuroscience Methods.

$515 \quad 2016 ; 264: 40-6$. 
25. Lesanpezeshki L, Hewitt JE, Laranjeiro R, Antebi A, Driscoll M, Szewczyk NJ, et al.

517 Pluronic gel-based burrowing assay for rapid assessment of neuromuscular health in C. elegans.

518 Scientific Reports. 2019;(1):15246. doi: 10.1038/s41598-019-51608-9.

519 26. Krajacic P, Shen X, Purohit PK, Arratia P, Lamitina T. Biomechanical profiling of

520 Caenorhabditis elegans motility. Genetics. 2012;191(3):1015-21.

521 27. Yemini E, Jucikas T, Grundy LJ, Brown AEX, Schafer WR. A database of

522 Caenorhabditis elegans behavioral phenotypes. Nature Methods. 2013;10(9):877-9.

523 28. Fang-Yen C, Wyart M, Xie J, Kawai R, Kodger T, Chen S, et al. Biomechanical analysis 524 of gait adaptation in the nematode Caenorhabditis elegans. Proceedings of the National Academy 525 of Sciences. 2010;107(47):20323-8.

526 29. Shen XN, Sznitman J, Krajacic P, Lamitina T, Arratia PE. Undulatory locomotion of 527 Caenorhabditis elegans on wet surfaces. Biophysical journal. 2012;102(12):2772-81.

528 30. Bilbao A, Patel AK, Rahman M, Vanapalli SA, Blawzdziewicz J. Roll maneuvers are 529 essential for active reorientation of Caenorhabditis elegans in 3D media. Proceedings of the 530 National Academy of Sciences. 2018:201706754.

531 31. Beron C, Vidal-Gadea AG, Cohn J, Parikh A, Hwang G, Pierce-Shimomura JT. The 532 burrowing behavior of the nematode Caenorhabditis elegans: a new assay for the study of 533 neuromuscular disorders. Genes, Brain and Behavior. 2015;14(4):357-68.

534 32. Johari S, Nock V, Alkaisi MM, Wang W. On-chip analysis of C. elegans muscular forces 535 and locomotion patterns in microstructured environments. Lab on a Chip. 2013;13(9):1699-707. micropillar arrays for high throughput measurements of forces applied by genetic model organisms. Biomicrofluidics. 2015;9(1):014111.

539 34. Rahman M, Hewitt J, Van-Bussel F, Edwards H, Blawzdziewicz J, Szewczyk N, et al. 540 NemaFlex: A microfluidics-based technology for standardized measurement of muscular 541 strength of C. elegans. Lab on a Chip. 2018;18(15):2187-201. doi: 10.1039/C8LC00103K.

542 35. Restif C, Ibáñez-Ventoso C, Vora MM, Guo S, Metaxas D, Driscoll M. CeleST: 543 computer vision software for quantitative analysis of $C$. elegans swim behavior reveals novel 544 features of locomotion. PLOS Computational Biology. 2014.

545 36. Ibáñez-Ventoso C, Herrera C, Chen E, Motto D, Driscoll M. Automated Analysis of $C$. 546 elegans Swim Behavior Using CeleST Software. JoVE (Journal of Visualized Experiments). 547 2016;(118):e54359-e.

548 37. Hewitt JE, Pollard AK, Lesanpezeshki L, Deane CS, Gaffney CJ, Etheridge T, et al. 549 Muscle strength deficiency and mitochondrial dysfunction in a muscular dystrophy model of 
550 Caenorhabditis elegans and its functional response to drugs. Disease Models \& Mechanisms.

$551 \quad 2018 ; 11(12): \mathrm{dmm} 036137$.

552 38. Laranjeiro R, Harinath G, Burke D, Braeckman BP, Driscoll M. Single swim sessions in

553 C. elegans induce key features of mammalian exercise. BMC Biology. 2017;15(1):30. doi:

554 10.1186/s12915-017-0368-4.

555 39. DeSilva S, Drachman DB, Mellits D, Kuncl RW. Prednisone treatment in Duchenne muscular dystrophy: long-term benefit. Archives of Neurology. 1987;44(8):818-22.

557 40. Chahbouni M, Escames G, Venegas C, Sevilla B, García JA, Lopez LC, et al. Melatonin treatment normalizes plasma pro-inflammatory cytokines and nitrosative/oxidative stress in patients suffering from Duchenne muscular dystrophy. Journal of Pineal Research. 2010;48(3):282-9.

561 41. Carre-Pierrat M, Mariol M-C, Chambonnier L, Laugraud A, Heskia F, Giacomotto J, et 562 al. Blocking of striated muscle degeneration by serotonin in C. elegans. Journal of Muscle 563 Research \& Cell Motility. 2006;27(3-4):253-8. Histamine Therapy Increases Tetanic Forces of Myoblasts, Reduces Muscle Injury, and Improves Grip Strength Performance of Dmdmdx Mice. Dose-Response.

$567 \quad 2015 ; 13(4): 1559325815616351$.

43. Hughes KJ, Rodriguez A, Flatt KM, Ray S, Schuler A, Rodemoyer B, et al. Physical exertion exacerbates decline in the musculature of an animal model of Duchenne muscular dystrophy. Proceedings of the National Academy of Sciences. 2019;116(9):3508-17.

44. Ghosh R, Mohammadi A, Kruglyak L, Ryu WS. Multiparameter behavioral profiling reveals distinct thermal response regimes in Caenorhabditis elegans. BMC Biology. 2012;10(1):85.

45. Feng Z, Cronin CJ, Wittig JH, Sternberg PW, Schafer WR. An imaging system for standardized quantitative analysis of $C$. elegans behavior. BMC Bioinformatics. 2004;5(1):115.

46. Nahabedian JF, Qadota H, Stirman JN, Lu H, Benian GM. Bending amplitude-A new quantitative assay of $C$. elegans locomotion: Identification of phenotypes for mutants in genes encoding muscle focal adhesion components. Methods. 2012;56(1):95-102.

47. Oh KH, Kim H. Reduced IGF signaling prevents muscle cell death in a Caenorhabditis elegans model of muscular dystrophy. Proceedings of the National Academy of Sciences. 2013;110(47):19024-9.

48. Yuan J, Ko H, Raizen DM, Bau HH. Terrain following and applications: Caenorhabditis elegans swims along the floor using a bump and undulate strategy. Journal of The Royal Society Interface. 2016;13(124):20160612. 
585 49. Sznitman J, Purohit PK, Krajacic P, Lamitina T, Arratia PE. Material properties of

586 Caenorhabditis elegans swimming at low Reynolds number. Biophysical Journal.

587 2010;98(4):617-26.

588 50. Gaud A, Simon J-M, Witzel T, Carre-Pierrat M, Wermuth CG, Ségalat L. Prednisone

589 reduces muscle degeneration in dystrophin-deficient Caenorhabditis elegans. Neuromuscular

590 Disorders. 2004;14(6):365-70.

591 51. Laranjeiro R, Harinath G, Hewitt JE, Hartman JH, Royal MA, Meyer JN, et al. Swim 592 exercise in Caenorhabditis elegans extends neuromuscular and gut healthspan, enhances learning 593 ability, and protects against neurodegeneration. Proceedings of the National Academy of 594 Sciences. 2019;116(47):23829-39.

595 52. Livak KJ, Schmittgen TD. Analysis of relative gene expression data using real-time 596 quantitative PCR and the 2- $\Delta \Delta$ CT method. Methods. 2001;25(4):402-8.

597 53. Hoogewijs D, Houthoofd K, Matthijssens F, Vandesompele J, Vanfleteren JR. Selection 598 and validation of a set of reliable reference genes for quantitative sod gene expression analysis in 599 C. elegans. BMC Molecular Biology. 2008;9(1):9. 